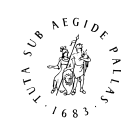

B R I L L

\title{
Touching Curvature and Feeling Size: a Contrast Illusion
}

\author{
Myrthe A. Plaisier ${ }^{1,2, *}$ and Marc O. Ernst ${ }^{2}$ \\ ${ }^{1}$ Faculty of Human Movement Sciences, VU University, van der Boechoststraat 9 , \\ 1081 BT Amsterdam, The Netherlands \\ ${ }^{2}$ Cognitive Neurosciences Department, Bielefeld University, Bielefeld, Germany
}

Received 15 July 2013; accepted 2 October 2013

\begin{abstract}
We know that our eyes can be deceiving. Here we demonstrate that we should not always trust our sense of touch either. Previous studies have shown that when pinching an object between thumb and index finger, we can under many circumstances accurately perceive its size. In contrast, the current results show that the local curvature at the areas of contact between the object and the fingers causes systematic under- or overestimation of the object's size. This is rather surprising given that local curvature is not directly related to the object's size. We suggest an explanation in terms of a contrast between the finger separation and an inferred relationship between local curvature and size. This study provides the first demonstration of an illusory haptic size percept caused by local curvature in a pinch grip.
\end{abstract}

\section{Keywords}

Tactile, proprioception, size illusion

\section{Introduction}

While the physical size of an object is constant, the perceived size of this object may vary depending on how we explore the object (Teghtsoonian and Teghtsoonian, 1965, 1970). For a pinch grip between the thumb and the index finger, it has been shown that perceived size of an object does not vary with its orientation in space (Gepshtein and Banks, 2003). Furthermore, variables such as the squeezing force, material compressibility, and contact area do not bias the size percept (Berryman et al., 2006). Berryman and collegues suggested that local object properties at the contact area with the fingers do not bias perceived size. However, this does not imply that cutaneous information

\footnotetext{
${ }^{*}$ To whom correspondence should be addressed. E-mail: M.A.Plaisier@vu.nl 
is irrelevant for size perception. For example, Berryman et al. (2006) hypothesized that perceived size is dominated by finger separation at the moment of contact with the object. The moment of contact is to a large degree signaled through cutaneous input.

To successfully grasp and manipulate objects in general, cutaneous inputs play an important role (e.g., Jenmalm and Johansson, 1997; Jenmalm et al., 2003; Johansson and Westling, 1984). There has been, however, no evidence of these local surface properties introducing a bias in the perceived size of the object. When pinching an object between two fingers only local information from the area of the object which is in contact with the finger pads is available. Local object information is not directly related to the object size and, therefore, might not be taken into account while judging size. To the contrary, in the case of local curvature there exist evidence that it plays a role in perception of global properties of objects such as size and orientation (Horst and Kappers, 2008; Panday et al., 2012). Local curvature, however, is not directly related to object size. So, why would this specific local property influence perceived size?

In the case of curvature, a relationship between local curvature and size may be inferred. For instance, for a circle there is a direct relationship between size and curvature. If we assume that the object being touched is circular, the local curvature perceived at the finger pads may carry information about the object size. In visual perception it has been demonstrated that participants assume objects to be predominantly circular (e.g. Knill, 2007; Muller et al., 2009). If a similar assumption plays a role in touch, we would expect local curvature to influence perceived size using a pinch grip. We might also expect that the size percept can be biased by local curvature. If a circular shape is indeed assumed then a conflict may arise between size information from finger separation and the inferred size from local curvature at the contact with the finger pad. For instance, for an elliptical object the local curvature would be high when pinching the object along the major axis. This curvature would correspond to a circle with a much smaller diameter than the major axis of the ellipse. Hence, a conflict would arise between the perceived size from finger separation and the size inferred from curvature.

Recently, we have found evidence suggesting that local curvature influences perceived size (Plaisier and Ernst, 2013). Here we further systematically investigate this bias. We asked participants to compare the diameter of a circular reference shape to the major and minor axes of ellipses with varying aspect ratios. For minor axis comparisons local curvature would indicate a circular object with a larger diameter than the size of the minor axis of the ellipse and vice versa for major axis comparisons. If a circularity assumption (or circularity prior) plays a role here, we would expect that major and minor axis judgments to be biased in opposite directions. 


\section{Methods}

Ten students (mean age 21, 3 left-handed) of Bielefeld University participated in the study. They were treated in accordance with the local guidelines and received payment for their participation. The project was approved by the local ethics committee. Participants were blindfolded and seated in front of a table. Stimuli consisted of 2D circles and ellipses with varying aspect ratios made out of Plexiglas. The shapes were mounted such that they could be pinched between thumb and index finger, but the shapes were fixed to the table such that they could not be moved, rotated, or lifted. The two reference shapes were circular (diameters 2.6 and $4.6 \mathrm{~cm}$ ) whereas the comparison shapes were ellipsoids with major or minor axes that were the same as the diameter of the reference circles. The reference and comparison shapes were always presented simultaneously and participants pinched one shape with the left hand and the other with the right hand. Participants were allowed to make small exploratory movements around the presented axis of the ellipse $\left(<20^{\circ}\right)$ but were explicitly instructed not to trace the circumference of the shape. The experimenter observed the participant during exploration to ensure this. The orientation of the pinch grip was parallel to the sagittal plane. After each trial the participant indicated which of the two shapes felt longer. Major and minor axes comparisons were presented randomly interleaved in a single block of trials to prevent any learning effects. Each reference-comparison pair was presented ten times. Five times the reference was presented to the left hand and the comparison to the right hand and five times vice versa. Furthermore, ten catch trials were included where an actual size difference existed between the reference and the comparison. In the catch trials, the $4.6 \mathrm{~cm}$ diameter reference was compared to an ellipsoid with major axis of $3.8 \mathrm{~cm}$. These trials were included to ensure that it was size that was being judged. Participants correctly identified the reference as being larger in $99 \%$ of the trials.

\section{Results}

In Fig. 1a it can be seen that the minor axis was always underestimated compared to the circular reference (Wilcoxon signed rank test, $p=0.003$ ), whereas the length of the major axis was always overestimated with respect to the circular reference (Wilcoxon signed rank test, $p=0.01$ ). The proportion of trials in which the reference was judged as being larger is shown as a function of aspect ratio in Fig. 1b. Although the major and minor axes comparisons were related to different diameters, when plotted on the same aspect ratio axis they follow a continuous trend. For minor axes comparisons as well as for major axes comparisons, there was an effect of aspect ratio difference (Friedman test: $\chi^{2}(4,36)=14, p=0.007$ and $\chi^{2}(5,45)=15, p=0.01$, respectively). 

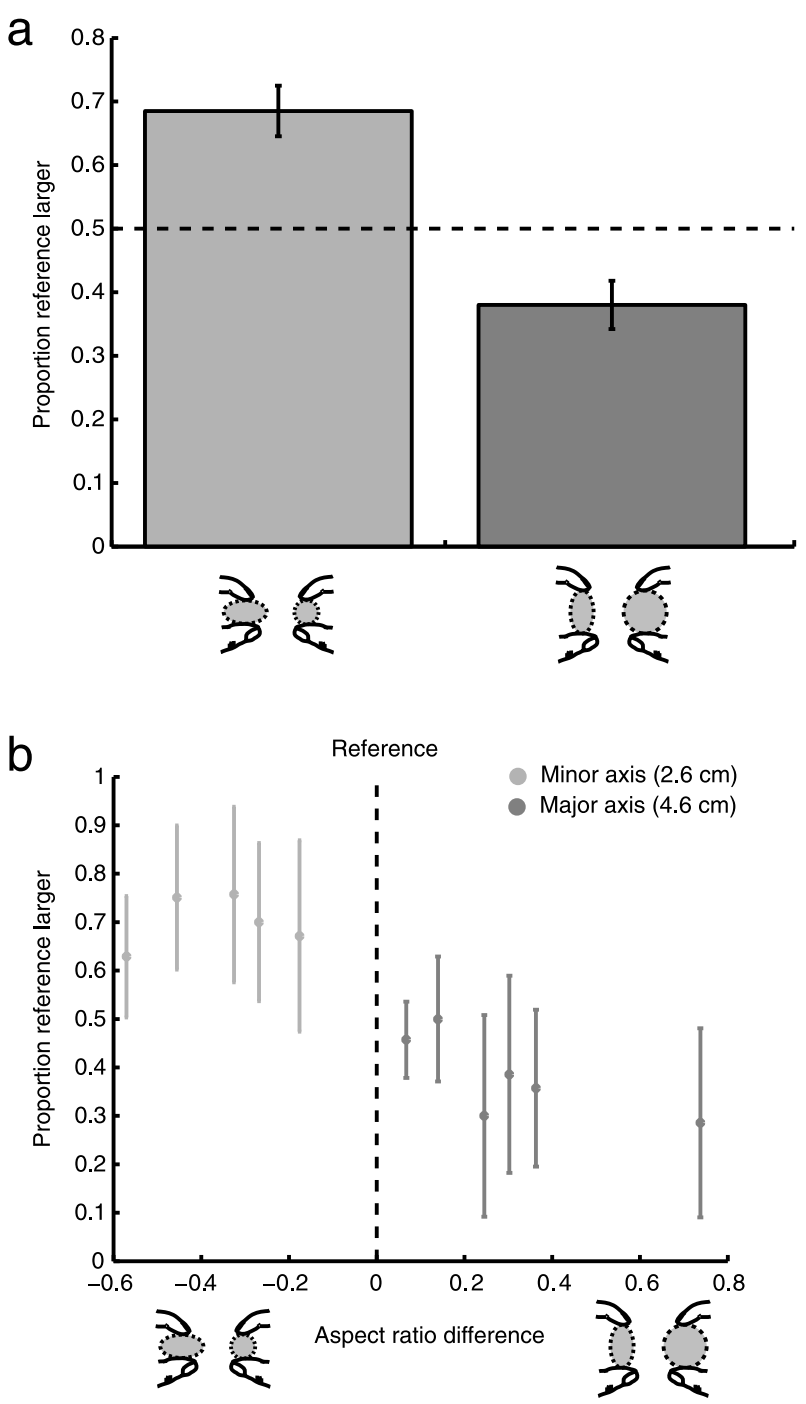

Figure 1. (a) Categorical means of minor and major axis comparisons averaged over participants. The dashed line indicates chance level and error bars indicate the between subjects standard error (SE). (b) Proportion of reference-larger responses as a function of the aspect ratio plotted on a log scale. Aspect ratio was calculated as the length of the axis that had to be estimated divided by the length of the other axis. This means that negative aspect ratio differences relate to minor axis comparisons and positive ones to major axis comparisons. Error bars indicate the between subjects standard deviation (SD).

\section{Discussion}

Our results show that the perceived size of an object using a pinch grip is influenced by the local curvature of an object at the points of contact with the 
finger pads. More specifically, the diameter of an ellipse pinched along the major axis was underestimated compared to the diameter of a circle. On the other hand, pinching along the minor axis lead to an underestimation of the diameter. These results support the hypothesis that a relationship is inferred between the local curvature at the points of contact and the object size. Several studies have shown that prior assumptions about object properties can influence the perceptual estimates of these properties (e.g. Adams et al., 2004; Ernst, 2007; Girshick et al., 2011; Knill, 2007; Weiss et al., 2002). In many cases this means that the perceptual estimate is influenced in the direction of the prior estimate.

Note that in the results presented here, the minor axis was underestimated, while the major axis was overestimated. If a participant has the prior assumption that objects are circular, giving rise to a relationship between size and local curvature, we would expect the size estimate to be biased towards the diameter of a circle that would correspond to the local curvature. For the major axis of an ellipse this would imply that this axis should actually be underestimated. We have, however, found the opposite effect. This can be interpreted as a repulsion of the perceptual estimate away from the prior estimate. One could interpret this as a two-stage process in which the size inferred from local curvature leads to a cue-conflict with the size indicated by finger separation. Whether the under- and overestimations stem from a prior that relates the local curvature to size or via a cue-conflict cannot be distinguished based on the current data. Either way, the illusion reported here shows similarities with the size-weight illusion where sensory discrepancy also seems to be enhanced (e.g. Brayanov and Smith, 2010; Ernst, 2009; Flanagan et al., 2008).

In the present study shapes were explored by actively pinching it between index finger and thumb. It could be that the illusion is smaller or absent if the shapes would be explored differently. For instance, by placing the finger first on one side of the object and subsequently moving to the other side of the object. In other haptic size illusions such as the horizontal-vertical size illusion or Müller-Lyer illusion, it has been shown that the size of these illusions indeed depends on the mode of exploration (Heller et al., 2005, 2010). In our experiment some exploratory differences were observed between participants. Some participants pinched the objects statically, while others preferred to move the fingers over the object while pinching. Regardless of these differences, all participants showed the illusion.

It has been previously shown that the presence of curvature can improve perceptual thresholds for size and orientation (Horst and Kappers, 2008; Panday et al., 2012). Here we have shown that when the shape is not circular, an illusory size is perceived. So, our results suggest that local curvature influences perceived size. We propose that this may be explained by an inferred relation- 
ship between curvature and size. The exact characteristics of this perceptual prior remain to be investigated.

\section{Acknowledgements}

This research was supported by a Rubicon grant from the Netherlands Organisation for Scientific Research (NWO) together with the Marie Curie Co-fund for MAP and by the Collaborative Project no. 248587, THE Hand Embodied.

\section{References}

Adams, W., Graf, E. and Ernst, M. (2004). Experience can change the 'light-from-above' prior, Nat. Neurosci. 7, 1057-1058.

Berryman, L., Yau, J. and Hsiao, S. (2006). Representation of object size in the somatosensory system, J. Neurophysiol. 96, 27-39.

Brayanov, J. B. and Smith, M. A. (2010). Bayesian and "Anti-Bayesian" biases in sensory integration for action and perception in the size-weight illusion, J. Neurophysiol. 103, 15181531.

Ernst, M. O. (2007). Learning to integrate arbitrary signals from vision and touch, J. Vis. 7(5), 7.

Ernst, M. O. (2009). Perceptual learning: Inverting the size-weight illusion, Curr. Biol. 19, R23-R25.

Flanagan, J. R., Bittner, J. P. and Johansson, R. S. (2008). Experience can change distinct sizeweight priors engaged in lifting objects and judging their weights, Curr. Biol. 8, 1742-1747.

Gepshtein, S. and Banks, M. (2003). Viewing geometry determines how vision and haptics combine in size perception, Curr. Biol. 13, 483-488.

Girshick, A. R., Landy, M. S. and Simoncelli, E. P. (2011). Cardinal rules: Visual orientation perception reflects knowledge of environmental statistics, Nat. Neurosci. 14, 926-932.

Heller, M. A., McCarthy, M., Schultz, J., Greene, J., Shanley, M., Clark, A., Skoczylas, S. and Prociuk, J. (2005). The influence of exploration mode, orientation and configuration on the haptic Muller-Lyer illusion, Perception 34, 1475-1500.

Heller, M. A., Walk, A. D. M., Schnarr, R., Kibble, S., Litwiller, B. and Ambuehl, C. (2010). Attenuating the haptic horizontal-vertical curvature illusion, Atten. Percept. Psychophys. 72, 1626-1641.

Jenmalm, P., Birznieks, I., Goodwin, A. W. and Johansson, R. S. (2003). Influence of object shape on responses of human tactile afferents under conditions characteristic of manipulation, Eur. J. Neurosci. 18, 164-176.

Jenmalm, P. and Johansson, R. (1997). Visual and somatosensory information about object shape control manipulative fingertip forces, J. Neurosci. 17, 4486-4499.

Johansson, R. and Westling, G. (1984). Roles of glabrous skin receptors and sensorimotor memory in automatic control of precision grip when lifting rougher or more slippery objects, Exp. Brain Res. 56, 550-564.

Knill, D. C. (2007). Learning Bayesian priors for depth perception, J. Vis. 7(8), 13.

Muller, C. M. P., Brenner, E. and Smeets, J. B. J. (2009). Maybe they are all circles: Clues and cues, J. Vis. 9(9), 10. 
Panday, V., Tiest, W. M. B. and Kappers, A. M. L. (2012). Influence of local properties on the perception of global object orientation, IEEE Trans. Haptics 5, 58-65.

Plaisier, M. A. and Ernst, M. O. (2013). Introducing the shape-length illusion, in: World Haptics Conference (WHC), 2013, Daejeon, Korea, pp. 583-586, IEEE.

Teghtsoonian, M. and Teghtsoonian, R. (1965). Seen and felt length, Psychon. Sci. 3, 465-466.

Teghtsoonian, R. and Teghtsoonian, M. (1970). Two varieties of perceived length, Percept. Psychophys. 8, 389-392.

van der Horst, B. J. and Kappers, A. M. L. (2008). Using curvature information in haptic shape perception of 3D objects, Exp. Brain Res. 190, 361-367.

Weiss, Y., Simoncelli, E. and Adelson, E. (2002). Motion illusions as optimal percepts, Nat. Neurosci. 5, 598-604. 